\title{
Fractional Integrals and Riesz Transforms Acting on Certain Lipschitz Spaces
}

\author{
M. Ramseyer, O. Salinas, \& B. Viviani
}

Abstract. We make a unifying approach to the study of mapping properties of fractional integrals and Riesz transforms acting on spaces of functions $f$ satisfying

$$
\sup _{B}\left(\frac{1}{w(a, r)}\left(\frac{1}{|B|} \int_{B}\left|f-m_{B} f\right|^{q}\right)^{1 / q}\right)<\infty,
$$

where $w$ is a nonnegative functional defined on the family of balls $B \subset \mathbb{R}^{n}$ with center $a$ and radius $r$. So, at the same time, we are able to treat such cases as BMO, Lipschitz spaces, and spaces of functions with variable smoothness among others. Results about pointwise smoothness related to these spaces are included as well.

\section{Introduction}

Let $w: \mathbb{R}^{n} \times \mathbb{R}_{+} \rightarrow \mathbb{R}_{+}$be a measurable function. For given $1 \leq q<\infty$, we define the space $\mathrm{BMO}_{w, q}$ as the set of locally integrable functions $f$ on $\mathbb{R}^{n}$ such that

$$
\frac{1}{w(a, r)}\left(\frac{1}{|B|} \int_{B}\left|f(x)-m_{B} f\right|^{q} d x\right)^{1 / q} \leq C
$$

for some $C>0$ and for every ball $B \subset \mathbb{R}^{n}$ with center $a$ and radius $r$, where $m_{B} f$ is the average of $f$ over $B$, namely $m_{B} f=|B|^{-1} \int_{B} f(y) d y$. As it can be easily seen, the expression

$$
\|f\|_{w, q}=\sup _{B \subset \mathbb{R}^{n}}\left\{\frac{1}{w(a, r)}\left(\frac{1}{|B|} \int_{B}\left|f(x)-m_{B} f\right|^{q} d x\right)^{1 / q}\right\}
$$

turns out to be a seminorm for this space. Then, $\mathrm{BMO}_{w, q}$ modulo constants is a Banach space. The space $\mathrm{BMO}_{w, 1}$ was introduced by Nakai and Yabuta [19], although a version defined on the $n$-dimensional torus had already appeared in Janson [14] in connection with the identification of pointwise multipliers of the space of functions with mean oscillation controlled by a positive, nondecreasing function $\varphi$, that is, $\mathrm{BMO}_{\varphi}$ (see [23]). The general $\mathrm{BMO}_{w, q}, 1 \leq q<\infty$, was introduced in [20], where a complete study on their pointwise multipliers is done.

Received May 1, 2014. Revision received October 8, 2015.

This research is partially supported by grants from Consejo Nacional de Investigaciones Científicas y Técnicas (CONICET) and Facultad de Ingeniería Química Universidad Nacional del Litoral (UNL), Argentina. 
As in [20], we suppose the following properties of $w$. We assume that there exists a positive constant $C$ such that

$$
\begin{aligned}
w\left(x, t_{1}\right) & \leq C w\left(x, t_{2}\right), \quad \forall x \in \mathbb{R}^{n}, \forall t_{1}<t_{2} . \\
w(x, 2 t) & \leq C w(x, t), \quad \forall x \in \mathbb{R}^{n}, \forall t>0 . \\
|x-y| & <t \Rightarrow w(x, t) \leq C w(y, t), \quad \forall x, y \in \mathbb{R}^{n}, \forall t>0 .
\end{aligned}
$$

As a first remark, since $w(x, t)$ satisfies (1.2) and (1.3), the definition of $\mathrm{BMO}_{w, q}$ through inequality (1.1) over cubes with center $a$ and sidelength $r$ instead balls is clearly equivalent. On the other hand, we say that the one-variable function $w(x, \cdot)$ satisfies the doubling condition if (1.3) holds for each $x$.

The spaces $\mathrm{BMO}_{w, q}$ provide an adequate setting to make a unifying approach to the study of several well-known spaces. For instance, a particular case of (1.1) can be found in [17], where the authors prove a weighted extension of the result that the Hilbert transform is a bounded map of $L^{\infty}$ into BMO. Also, taking $q=1$ and $w(x, t)=\Phi(t) t^{-n} \int_{B(x, t)} v(y) d y$ with a positive and locally integrable function $v$ and assuming certain properties on $\Phi: \mathbb{R}^{+} \rightarrow \mathbb{R}^{+}$, we get the $\operatorname{BMO}_{\Phi}(v)$ of [13]. For $\Phi(t)=t^{n}$, we recover the weighted BMO space of Muckenhoupt and Wheeden [18]. When $v \equiv 1$, we get the classical BMO $(\Phi \equiv 1)$, the Lipschitz integral spaces $\left(\Phi(t)=t^{\beta}, \beta>0\right)$, and, for a more general $\phi$, the spaces $\mathrm{BMO}_{\Phi}$ considered by Spanne [23]. (See [3] and [16] in addition.)

The case $w(x, t)=t^{\alpha-n}\left\|\chi_{B(x, t)}\right\|_{p^{\prime}(\cdot)}$, where $0<\alpha<n, p^{\prime}(\cdot)=p(\cdot) /(p(\cdot)-$ $1)$, and $\|\cdot\|_{p(\cdot)}$ denotes the norm in the variable Lebesgue space $L^{p(\cdot)}$ (see [15]), has a special interest since the spaces $\mathrm{BMO}_{w, q}$ are the spaces $\mathfrak{L}_{\alpha, p(\cdot)}^{q}$ introduced in [22], which, under a natural condition on $p(\cdot)$, turn out to coincide with $\mathfrak{L}_{\alpha, p(\cdot)}$ (see Corollary 2.22). In turn, the space $\mathfrak{L}_{\alpha, p(\cdot)}$ has been identified (see Thms. 1.11 and 1.13 in [22]) as a suitable target space for the fractional integral operator acting on certain $L^{p(\cdot)}$.

Some particular cases of the spaces (1.1) are useful in the study of regularity of solutions of elliptic PDEs (see, e.g., [1; 2], and [22]).

The main purpose of our article is to make a unifying approach to the study of mapping properties of fractional integrals and Riesz transforms in relation to the spaces $\mathrm{BMO}_{w, q}$, so that this approach includes all the aforementioned particular cases. In addition, we prove some properties of these spaces such as, for instance, a pointwise characterization.

The structure of the article is as follows. Section 2 contains properties of the $\mathrm{BMO}_{w, q}$ spaces in general (Section 2.1) and the particular case $\mathfrak{L}_{\alpha, p(\cdot)}$ (Section 2.2). In Section 3 we present our main results related to the boundedness of the fractional integral. Finally, Section 4 is devoted to the boundedness of Riesz transforms.

\section{Properties of the Spaces $\mathbf{B M O}_{w, q}$ and $\mathfrak{L}_{\alpha, p(\cdot)}^{q}$}

In this section we prove some useful properties of the spaces involved. We start by recalling some definitions and properties related to real functions. They will be important tools in our results. 
Definition 2.1. Let $h: \mathbb{R}_{0}^{+} \rightarrow \mathbb{R}_{0}^{+}$be a function. We say that $h$ is of upper type $\beta>0$ if there exists a positive constant $c$ such that

$$
h(s t) \leq c s^{\beta} h(t)
$$

for all $s \geq 1$ and $t>0$. We also say that $h$ is of lower type $\beta>0$ if the last inequality holds for all $0<s \leq 1$ and $t>0$. We say that $h$ satisfies the doubling condition if there exists a constant $c$ such that $h(2 t) \leq c h(t)$ for all $t>0$.

Definition 2.2. We say that $h$ is quasi-decreasing if there exists a constant $c$ such that $h\left(t_{2}\right) \leq \operatorname{ch}\left(t_{1}\right)$ whenever $t_{1}<t_{2}$.

The proofs of the following lemmas are easy and left to the reader.

Lemma 2.3. Let $h$ be a function of upper type $\beta$ with $0<\beta \leq 1$. Then $h$ satisfies the doubling condition. Moreover, $h(t) / t$ is quasi-decreasing.

LEMma 2.4. Consider a function $h$ such that $h(t) / t^{\beta}$ is quasi-decreasing for some $0<\beta \leq 1$. Then $h$ is of upper type $\beta$. Moreover, $h$ satisfies the doubling condition.

\subsection{The Space $\mathrm{BMO}_{w, q}$}

Now we study conditions on the function $w: \mathbb{R}^{n} \times \mathbb{R}_{+} \rightarrow \mathbb{R}_{+}$under which the functions belonging to $\mathrm{BMO}_{w, q}$ satisfy some kind of pointwise smoothness. Conversely, we also see that, under certain hypothesis on $w$, this smoothness implies that $w$ belongs to $\mathrm{BMO}_{w, q}$.

Proposition 2.5 (Pointwise condition). Let $1 \leq q<\infty$, and let $w(x, t)$ be a function satisfying (1.2). Then, for every $f \in B M O_{w, q}$, we have

$$
|f(x)-f(y)| \leq C\|f\|_{w, q} \int_{0}^{4|x-y|}(w(x, t)+w(y, t)) \frac{d t}{t}
$$

for some constant $C>0$ and for almost all $x, y \in \mathbb{R}^{n}$.

Proof. Let $x, y$ be Lebesgue points of $f$ in $\mathbb{R}^{n}$. Taking $B=B(x,|x-y|)$ and $B^{\prime}=B(y,|x-y|)$, we have

$$
|f(x)-f(y)| \leq\left|f(x)-m_{B} f\right|+\left|f(y)-m_{B^{\prime}} f\right|+\left|m_{B^{\prime}} f-m_{B} f\right| .
$$

We only estimate the first term on the right-hand side since the second is similar. Letting $B_{i}=B\left(x, 2^{-i}|x-y|\right)$ for each integer $i$ and using the hypothesis on $w$, we get

$$
\begin{aligned}
\left|f(x)-m_{B} f\right| & \leq \lim _{k \rightarrow \infty}\left(\left|f(x)-m_{B_{k}} f\right|+\sum_{i=0}^{k-1}\left|m_{B_{i+1}} f-m_{B_{i}} f\right|\right) \\
& \leq C \sum_{i=0}^{\infty}\left(\left|B_{i}\right|^{-1} \int_{B_{i}}\left|f(z)-m_{B_{i}} f\right|^{q} d z\right)^{1 / q}
\end{aligned}
$$




$$
\begin{aligned}
& \leq C\|f\|_{w, q} \sum_{i=0}^{\infty} w\left(x, 2^{-i}|x-y|\right) \\
& =C\|f\|_{w, q} \sum_{i=0}^{\infty} \int_{2^{-i}|x-y|}^{2^{-i+1}|x-y|} w\left(x, 2^{-i}|x-y|\right) \frac{d t}{t} \\
& \leq C\|f\|_{w, q} \int_{0}^{2|x-y|} w(x, t) \frac{d t}{t} .
\end{aligned}
$$

Finally, denoting $2 B=B(x, 2|x-y|)$, we have

$$
\begin{aligned}
\left|m_{B^{\prime}} f-m_{B} f\right| & \leq\left|m_{B^{\prime}} f-m_{2 B} f\right|+\left|m_{2 B} f-m_{B} f\right| \\
& \leq C\|f\|_{w, q} \int_{0}^{4|x-y|} w(x, t) \frac{d t}{t} .
\end{aligned}
$$

This completes the proof.

REMARK 2.6. If, in addition, $w(x, t)$ satisfies the doubling condition (1.3), then we obtain

$$
|f(x)-f(y)| \leq C\|f\|_{w, q} \int_{0}^{|x-y|}(w(x, t)+w(y, t)) \frac{d t}{t}
$$

for almost all $x, y \in \mathbb{R}^{n}$.

Proposition 2.7. Let $w$ be a measurable function satisfying (1.3). Suppose that, for some $1 \leq q<\infty$,

$$
\Psi_{q}(x, r) \doteq\left(\frac{1}{r^{n}} \int_{B(x, r)}\left(\int_{0}^{r} w(z, t) \frac{d t}{t}\right)^{q} d z\right)^{1 / q}
$$

is finite for all $x \in \mathbb{R}^{n}$ and $r>0$. If a measurable function $f$ satisfies the pointwise condition (2.1), then $f \in B M O_{\Psi_{q}, q}$. Moreover, if there exists a constant $C>0$, independent of $x$ and $r$, such that

$$
\Psi_{q}(x, r) \leq C w(x, r),
$$

then $f \in B M O_{w, q}$.

Proof. The finiteness of $\Psi_{q}(x, r)$ implies that the right-hand side of (2.1) is finite a.e. Moreover, it is not difficult to see that $f$ is locally integrable. In order to prove that $f \in \mathrm{BMO}_{\Psi_{q}, q}$, we will prove that

$$
\int_{B}\left|f(y)-m_{B} f\right|^{q} d y \leq C \Psi_{q}(x, r)^{q}|B|
$$

for every ball $B=B(x, r)$. In fact,

$$
\begin{aligned}
\int_{B} \mid & f(y)-\left.m_{B} f\right|^{q} d y \\
& \leq C \int_{B}\left(\frac{1}{|B|} \int_{B}\left|\int_{0}^{4|y-z|}(w(y, t)+w(z, t)) \frac{d t}{t}\right| d z\right)^{q} d y
\end{aligned}
$$




$$
\begin{aligned}
& \leq C \int_{B}\left(\int_{0}^{8 r} w(y, t) \frac{d t}{t}+\frac{1}{|B|} \int_{B} \int_{0}^{8 r} w(z, t) \frac{d t}{t} d z\right)^{q} d y \\
& \leq C \int_{B}\left(\int_{0}^{8 r} w(y, t) \frac{d t}{t}\right)^{q} d y+C|B|^{1-q}\left(\int_{B} \int_{0}^{8 r} w(z, t) \frac{d t}{t} d z\right)^{q} \\
& \leq C \int_{B}\left(\int_{0}^{8 r} \frac{w(z, t)}{t} d t\right)^{q} d z
\end{aligned}
$$

where in the last step the Hölder inequality was applied. Thus, using the doubling condition on $w$, we have (2.5). Moreover, if (2.4) holds, then it is clear that $f \in$ $\mathrm{BMO}_{w, q}$, and the proposition is proved.

Propositions 2.5 and 2.7 allow us to get the following theorem.

THEOREM 2.8. Let $w$ be a measurable function satisfying (1.2) and (1.3). Moreover, suppose that (2.4) holds for some $1 \leq q<\infty$. Then $B M O_{w, 1}=B M O_{w, s}$ for every $1 \leq s \leq q$.

Proof. By Hölder's inequality it is clear that $\mathrm{BMO}_{w, s} \subset \mathrm{BMO}_{w, 1}$. On the other hand, if $f \in \mathrm{BMO}_{w, 1}$, then by Proposition $2.5 f$ satisfies (2.1). In view of Proposition 2.7, we have that $f \in \mathrm{BMO}_{w, q}$. Hence, again by Hölder's inequality, the theorem follows.

The next proposition gives sufficient conditions on $w$ for inequality (2.4).

Proposition 2.9. Let $w$ be a measurable function. If $w$ is of lower type $\beta>0$ on the second variable, then inequality (2.4) holds for every $1 \leq q<\infty$.

Proof. Let $B=B(x, r)$. Then by a change of variable we have

$$
\begin{aligned}
\int_{B}\left(\int_{0}^{r} \frac{w(z, u)}{u} d u\right)^{q} d z & =\int_{B}\left(\int_{0}^{1} \frac{w(z, r t)}{t} d t\right)^{q} d z \\
& \leq C \int_{B}\left(\int_{0}^{1} t^{\beta-1} d t\right)^{q} w(z, r)^{q} d z \\
& \leq C \int_{B} w(x, r)^{q} d z \leq C w(x, r)^{q} r^{n}
\end{aligned}
$$

This completes the proof.

We note that Theorem 2.8 uses the pointwise condition (2.1) and hypothesis (2.4). However, in the case $w \equiv 1$, neither of them is valid, and it is well known that $\mathrm{BMO}_{1,1}=\mathrm{BMO}_{1, q}$ for every $1 \leq q<\infty$. So it is natural to wonder what other properties of $w$ can assure the same coincidence of spaces. In order to give an answer, we first state the following result of Franchi, Pérez, and Wheeden [10].

Definition 2.10. For a number $1 \leq t<\infty$, we say that a function $w$ satisfies the $D_{t}$-condition if there exists a positive constant $c$ such that, for each ball $B=$ 
$B(x, r)$ and any family $\left\{B_{i}\right\}$ of pairwise disjoint subballs of $B$,

$$
\sum_{i} w\left(x_{i}, r_{i}\right)^{t} r_{i}^{n} \leq c^{t} w(x, r)^{t} r^{n}
$$

where $x_{i}$ and $r_{i}$ are the center and the radius of $B_{i}$, respectively. We denote by $\|w\|$ the smallest constant $c$ for which (2.6).

It is not difficult to see that the $D_{t}$-condition implies the $D_{s}$-condition for every $1 \leq s<t$

Theorem 2.11 ([10], Theorem 2.3). Let $B_{0}=B\left(x_{0}, r_{0}\right)$ be a ball in $\mathbb{R}^{n}$. Suppose that $w$ satisfies the $D_{t}$-condition for some $1 \leq t<\infty$. Let $f$ be a measurable function defined on $17 B_{0}$ and such that

$$
\frac{1}{|B|} \int_{B}\left|f(y)-f_{B}\right| d y \leq\|f\|_{w, 1} w(x, r)
$$

for every ball $B=B(x, r) \subset 17 B_{0}$. Then

$$
\sup _{\lambda>0} \lambda\left(\frac{\left|\left\{x \in B_{0}:\left|f-f_{B_{0}}\right|>\lambda\right\}\right|}{\left|B_{0}\right|}\right)^{1 / r} \leq C\|w\|\|f\|_{w, 1} w\left(x_{0}, 17 r_{0}\right),
$$

where the constant $C$ is independent of $f$ and $B_{0}$.

Corollary 2.12. Let $1<t<\infty$. Under the hypotheses of Theorem 2.11, we have

$$
\left(\frac{1}{\left|B_{0}\right|} \int_{B_{0}}\left|f(y)-f_{B_{0}}\right|^{q} d y\right)^{1 / q} \leq C\|w\|\|f\|_{w, 1} w\left(x_{0}, 17 r_{0}\right)
$$

for every $1<q<t$, where the constant $C$ is independent of $f$ and $B_{0}$.

Now, in view of Theorem 2.11 and its corollary, we are able to prove the following result.

THeOREM 2.13. Let $w$ be a measurable function satisfying (1.2), (1.3), and (1.4). Then, the spaces $B M O_{w, q}$ coincide for all $1 \leq q<\infty$.

Proof. It is easily seen that $\mathrm{BMO}_{w, q} \subset \mathrm{BMO}_{w}$. On the other hand, if $f \in \mathrm{BMO}_{w}$, then by Corollary 2.12 we have to see that $w$ satisfies the $D_{q}$-condition for every $1 \leq q<\infty$. In fact, let $B$ be a ball, and $\left\{B_{i}\right\}$ a family of pairwise disjoint subballs of $B$. Then, from the hypotheses on $w$ we have

$$
\begin{aligned}
\sum_{i} w\left(x_{i}, r_{i}\right)^{q} r_{i}^{n} & \leq C \sum_{i} w\left(x_{i}, r\right)^{q} r_{i}^{n} \leq C \sum_{i} w(x, r)^{q} r_{i}^{n} \\
& \leq C w(x, r)^{q} \sum_{i}\left|B_{i}\right| \leq C w(x, r)^{q}|B|=C w(x, r)^{q} r^{n}
\end{aligned}
$$

Then the spaces coincide. 


\subsection{The Space $\mathfrak{L}_{\alpha, p(\cdot)}^{q}$}

Here we consider the spaces $\mathfrak{L}_{\alpha, p(\cdot)}^{q}$ defined in the previous section. We denote by $p_{-}(\Omega)$ and $p_{+}(\Omega)$ the infimum and supremum of $p(\cdot)$ over a subset $\Omega$ of $\mathbb{R}^{n}$. We only write $p_{-}$and $p_{+}$in the case $\Omega=\mathbb{R}^{n}$. The following lemma shows very useful relations between the norm of a characteristic function of a ball and its Lebesgue measure.

LeMmA 2.14. Let $B=B\left(x_{0}, r\right)$ be a ball in $\mathbb{R}^{n}$.

(a) There exist positive constants $a_{1}$ and $a_{2}$ such that if $r<1$, then

$$
a_{1}|B|^{1 / p_{-}(B)} \leq\left\|\chi_{B}\right\|_{p(\cdot)} \leq a_{2}|B|^{1 / p_{+}(B)} .
$$

(b) There exist positive constants $b_{1}$ and $b_{2}$ such that if $r>1$, then

$$
b_{1}|B|^{1 / p_{+}(B)} \leq\left\|\chi_{B}\right\|_{p(\cdot)} \leq b_{2}|B|^{1 / p_{-}(B)} .
$$

In the setting of variable exponent Lebesgue spaces, it is common to assume the following log-Hölder conditions on the exponent functions $p(\cdot)$ :

$$
\begin{gathered}
L H_{0}: \quad \exists c_{0}>0 / \quad|p(x)-p(y)| \leq \frac{c_{0}}{\log (e+1 /|x-y|)}, \quad \forall x, y \in \mathbb{R}^{n} ; \\
L H_{\infty}: \quad \exists p_{\infty}, c_{1}>0 / \quad\left|p(x)-p_{\infty}\right| \leq \frac{c_{1}}{\log (e+|x|)}, \quad \forall x \in \mathbb{R}^{n} .
\end{gathered}
$$

REMARK 2.15. In [5], Proposition 4.57, it is proved that $L H_{0}$ and $L H_{\infty}$ imply that there exists a constant $C>0$ such that

$$
\left\|\chi_{B}\right\|_{p(\cdot)}\left\|\chi_{B}\right\|_{p^{\prime}(\cdot)} \leq C|B|
$$

for every ball $B \subset \mathbb{R}^{n}$. Considering this inequality and applying Hölder's inequality, we easily see that $w(x, \cdot)=\left\|\chi_{B(x, \cdot)}\right\|_{p(\cdot)}$ satisfies the following doubling condition:

$$
\left\|\chi_{B(x, 2 t)}\right\|_{p(\cdot)} \leq C\left\|\chi_{B(x, t)}\right\|_{p(\cdot)},
$$

where the constant $C$ is independent of $x$ and $t$. Obviously, $p^{\prime}(\cdot)$ has the same property.

Definition 2.16. If inequality (2.11) holds, then we say that the exponent function $p(\cdot)$ satisfies the doubling condition.

In connection with these log-Hölder continuity properties, we state two important lemmas, whose proofs can be found in several articles; see, for instance, $[9 ; 7 ; 11 ; 4]$.

Lemma 2.17. Let $p_{+}<\infty$. Then the following conditions are equivalent:

(a) The function $p(\cdot)$ satisfies $\mathrm{LH}_{0}$.

(b) There exists a constant $C$ such that

$$
|B|^{p_{-}(B)-p_{+}(B)} \leq C
$$

for every ball $B \subset \mathbb{R}^{n}$. 
LEMma 2.18. Let $p(\cdot)$ be an exponent function satisfying $\mathrm{LH}_{\infty}$. Then, there exists a constant $c$ such that

$$
c^{-1}|B|^{1 / p_{\infty}} \leq\left\|\chi_{B}\right\|_{p(\cdot)} \leq c|B|^{1 / p_{\infty}}
$$

for every ball $B$ with radius greater than or equal to $1 / 4$.

The following three technical lemmas give some properties of the particular function $w(x, t)=t^{\alpha-n}\left\|\chi_{B(x, t)}\right\|_{p^{\prime}(\cdot)}$.

LeMma 2.19. Let $p(\cdot)$ be an exponent function such that $p_{-} \geq \frac{n}{\alpha}$ and satisfying $L H_{0}$ and $L H_{\infty}$. Then $w(x, t)=t^{\alpha-n}\left\|\chi_{B(x, t)}\right\|_{p^{\prime}(\cdot)}$ is quasi-increasing as a function of $t$.

Proof. We will see that there exists a constant $C>0$ such that, given $0<s<t$, we have

$$
t^{\alpha-n}\left\|\chi_{B(x, t)}\right\|_{p^{\prime}(\cdot)} \leq C s^{\alpha-n}\left\|\chi_{B(x, s)}\right\|_{p^{\prime}(\cdot)},
$$

where $C$ does not depend on $x$. For this, we divide the proof into three parts.

(a) If $1<t<s$, by Lemma 2.18 and the hypotheses on $p(\cdot)$ we have

$$
\begin{aligned}
w(x, t) & =t^{\alpha-n}\left\|\chi_{B(x, t)}\right\|_{p^{\prime}(\cdot)} \leq C t^{\alpha-n} t^{n-n / p_{\infty}}=C t^{\alpha-n / p_{\infty}} \\
& \leq C s^{\alpha-n / p_{\infty}} \leq C s^{\alpha-n}\left\|\chi_{B(x, s)}\right\|_{p^{\prime}(\cdot)}=C w(x, s) .
\end{aligned}
$$

(b) Now, if $t<s<1$, then by Lemma 2.14 with $p^{\prime}(\cdot)$ instead of $p(\cdot)$ and Lemma 2.17, taking into account that $p_{-}(B(x, t)) \geq p_{-}(B(x, s))$, we have

$$
\begin{aligned}
w(x, t) & =t^{\alpha-n}\left\|\chi_{B(x, t)}\right\|_{p^{\prime}(\cdot)} \\
& \leq C t^{\alpha-n} t^{n-n / p_{-}(B(x, t))} \\
& \leq C s^{\alpha-n / p_{-}(B(x, t))} \\
& \leq C s^{\alpha-n / p_{+}(B(x, s))}\left(s^{n / p_{+}(B(x, s))-n / p_{-}(B(x, s))}\right) \\
& \leq C s^{\alpha-n}\left\|\chi_{B(x, s)}\right\|_{p^{\prime}(\cdot)}=C w(x, s) .
\end{aligned}
$$

(c) Finally, suppose that $t<1<s$. By Lemmas 2.14 and 2.18 we get that

$$
w(x, t) \leq C t^{\alpha-n / p_{-}(B(x, t))} \leq C \leq C s^{\alpha-n / p_{\infty}} \leq C w(x, s) .
$$

The proof is complete.

Lemma 2.20. Let $p(\cdot)$ be an exponent function satisfying $\mathrm{LH}_{0}$ and $\mathrm{LH}_{\infty}$. Then there exists $C>0$ such that, for all $t>0$,

$$
\left\|\chi_{B(x, t)}\right\|_{p^{\prime}(\cdot)} \leq C\left\|\chi_{B(y, t)}\right\|_{p^{\prime}(\cdot)}
$$

whenever $|x-y|<t$.

Proof. It is not difficult to see that $p^{\prime}(\cdot)$ satisfies $L H_{0}$ and $L H_{\infty}$ whenever $p(\cdot)$ does. Moreover, $1 / p_{\infty}^{\prime}=1-1 / p_{\infty}$. So, in order to prove the lemma, we are going to consider two cases. First, suppose $t>1$. By Lemma 2.18 we have

$$
\left\|\chi_{B(x, t)}\right\|_{p^{\prime}(\cdot)} \leq C|B(x, t)|^{1-1 / p_{\infty}}=C|B(y, t)|^{1-1 / p_{\infty}} \leq C\left\|\chi_{B(y, t)}\right\|_{p^{\prime}(\cdot)} .
$$


Now, suppose $t \leq 1$. Since $B(y, t) \subset B(x, 2 t)$ if $|x-y|<t$, by Lemmas 2.14 and 2.17 we have

$$
\begin{aligned}
\left\|\chi_{B(x, t)}\right\|_{p^{\prime}(\cdot)} & \leq C|B(x, t)|^{1-1 / p_{-}(B(x, t))} \\
& =C|B(x, t)|^{1-1 / p_{+}(B(y, t))}|B(x, t)|^{1 / p_{+}(B(y, t))-1 / p_{-}(B(x, t))} \\
& \leq C|B(y, t)|^{1-1 / p_{+}(B(y, t))}\left(|B(x, t)|^{p_{-}(B(x, 2 t))-p_{+}(B(x, 2 t))}\right)^{1 / p_{-}^{2}} \\
& \leq C\left\|\chi_{B(y, t)}\right\|_{p^{\prime}(\cdot)}
\end{aligned}
$$

and the lemma is proved.

Lemma 2.21. Let $p(\cdot)$ be an exponent function such that $p_{-}>\frac{n}{\alpha}$. If $p(\cdot)$ satisfies $\mathrm{LH}_{0}$ and $\mathrm{LH}_{\infty}$, then

$$
\int_{0}^{r} \frac{\left\|\chi_{B(x, t)}\right\|_{p^{\prime}(\cdot)}}{t^{n-\alpha}} \frac{d t}{t} \leq C \frac{\left\|\chi_{B(x, r)}\right\|_{p^{\prime}(\cdot)}}{r^{n-\alpha}}
$$

for every $B=B(x, r)$, where $C$ is independent of $B$.

Proof. Fix $x \in \mathbb{R}^{n}$. First, we suppose $r \leq 1$. By Lemma 2.14 we have

$$
\begin{aligned}
\int_{0}^{r} \frac{\left\|\chi_{B(x, t)}\right\|_{p^{\prime}(\cdot)}}{t^{n-\alpha}} \frac{d t}{t} & \leq C \int_{0}^{r} \frac{|B(x, t)|^{1-1 / p_{-}(B(x, t))}}{t^{n-\alpha}} \frac{d t}{t} \\
& \leq C \int_{0}^{r} t^{\alpha-n / p_{-}(B(x, r))-1} d t=C r^{\alpha-n / p_{-}(B(x, r))} .
\end{aligned}
$$

Now, again from Lemma 2.17 and Lemma 2.14 we get

$$
\begin{aligned}
\int_{0}^{r} \frac{\left\|\chi_{B(x, t)}\right\|_{p^{\prime}(\cdot)}}{t^{n-\alpha}} \frac{d t}{t} & \leq C r^{\alpha-n / p_{+}(B(x, r))}\left(r^{n / p_{+}(B(x, r))-n / p_{-}(B(x, r))}\right) \\
& \leq C \frac{\left\|\chi_{B(x, r)}\right\|_{p^{\prime}(\cdot)}}{r^{n-\alpha}} .
\end{aligned}
$$

On the other hand, if $r>1$, then

$$
\int_{0}^{r} \frac{\left\|\chi_{B(x, t)}\right\|_{p^{\prime}(\cdot)}}{t^{n-\alpha}} \frac{d t}{t}=\int_{0}^{1} \frac{\left\|\chi_{B(x, t)}\right\|_{p^{\prime}(\cdot)}}{t^{n-\alpha}} \frac{d t}{t}+\int_{1}^{r} \frac{\left\|\chi_{B(x, t)}\right\|_{p^{\prime}(\cdot)}}{t^{n-\alpha}} \frac{d t}{t} .
$$

The previous estimate allows us to obtain

$$
\int_{0}^{1} \frac{\left\|\chi_{B(x, t)}\right\|_{p^{\prime}(\cdot)}}{t^{n-\alpha}} \frac{d t}{t} \leq C .
$$

Now, since $\alpha p_{-}-n>0$ and $\left(p_{-}\right)^{\prime}=\left(p^{\prime}\right)_{+}$, it is not difficult to see that $(\alpha-$ $n)\left(p_{-}\right)^{\prime}+n \geq 1$. Then we get

$$
\begin{aligned}
1 & <r^{(\alpha-n)\left(p_{-}\right)^{\prime}+n}=C|B(x, r)| r^{(\alpha-n)\left(p_{-}\right)^{\prime}}=C|B(x, r)| r^{(\alpha-n)\left(p^{\prime}\right)_{+}} \\
& <C \int_{B(x, r)} r^{(\alpha-n) p^{\prime}(y)} d y=C \int_{\mathbb{R}^{n}}\left(\frac{\chi_{B(x, r)}}{r^{(n-\alpha)}}\right)^{p^{\prime}(y)} d y .
\end{aligned}
$$

So it follows that $r^{(n-\alpha)}$ is a lower bound for $\left\|\chi_{B(x, r)}\right\|_{p^{\prime}(\cdot)}$. Then

$$
\int_{0}^{1} \frac{\left\|\chi_{B(x, t)}\right\|_{p^{\prime}(\cdot)}}{t^{n-\alpha}} \frac{d t}{t} \leq C \frac{\left\|\chi_{B(x, r)}\right\|_{p^{\prime}(\cdot)}}{r^{n-\alpha}} .
$$


For the second term, by Lemma 2.18 the estimate is clear. In fact,

$$
\begin{aligned}
\int_{1}^{r} \frac{\left\|\chi_{B(x, t)}\right\|_{p^{\prime}(\cdot)}}{t^{n-\alpha}} \frac{d t}{t} & \leq C \int_{1}^{r} \frac{\left|B_{t}\right|^{1-1 / p_{\infty}}}{t^{n-\alpha}} \frac{d t}{t} \leq C \int_{1}^{r} t^{\alpha-n / p_{\infty}-1} d t \\
& \leq C r^{\alpha-n / p_{\infty}} \leq C \frac{\left\|\chi_{B(x, r)}\right\|_{p^{\prime}(\cdot)}}{r^{n-\alpha}}
\end{aligned}
$$

Finally, note that inequalities (2.14), (2.15), and (2.16) imply (2.13).

Corollary 2.22. Let $0<\alpha<n$, and let $p(\cdot)$ be an exponent function with $p_{-}>\frac{n}{\alpha}$ such that conditions $L_{0}$ and $L H_{\infty}$ hold. Then $\mathfrak{L}_{\alpha, p(\cdot)}=\mathfrak{L}_{\alpha, p(\cdot)}^{q}$ for $1 \leq q<\infty$.

Proof. By Hölder's inequality it is clear that $\mathfrak{L}_{\alpha, p(\cdot)}^{q} \subset \mathfrak{L}_{\alpha, p(\cdot)}$. On the other hand, from Lemma 2.19, the hypotheses on $p(\cdot)$, and Proposition 2.5 we have that every $f \in \mathfrak{L}_{\alpha, p(\cdot)}$ satisfies the pointwise inequality (2.1). Moreover, the right-hand side of this inequality is finite.

Now, for fixed $1<q<\infty$, from Lemmas 2.21 and 2.20 we have

$$
\begin{aligned}
\int_{B(x, r)}\left(\int_{0}^{r} \frac{\left\|\chi_{B(z, u)}\right\|_{p^{\prime}(\cdot)}}{u^{n-\alpha}} \frac{d u}{u}\right)^{q} d z & \leq C \int_{B(x, r)} \frac{\left\|\chi_{B(z, r)}\right\|_{p^{\prime}(\cdot)}^{q}}{r^{(n-\alpha) q}} d z \\
& \leq C\left(r^{\alpha-n}\left\|\chi_{B(x, r)}\right\|_{p^{\prime}(\cdot)}\right)^{q} r^{n},
\end{aligned}
$$

which states that (2.4) holds for $w(x, r)=r^{\alpha-n}\left\|\chi_{B(x, r)}\right\|_{p^{\prime}(\cdot)}$. Then, Remark 2.15 and Proposition 2.7 ensure that $f \in \mathfrak{L}_{\alpha, p(\cdot)}^{q}$, which finishes the proof.

Now, if we consider an exponent function $p(\cdot)$ that does not necessarily verify the log-Hölder conditions $L H_{0}$ and $L H_{\infty}$, a different approach can be adopted. However, a smaller range of $q$ is obtained. In order to do this, we first recall (see [15]) that we can write the representation of the norm given by

$$
\|f\|_{p(\cdot)} \approx \sup _{g:\|g\|_{p^{\prime}(\cdot)} \leq 1} \int_{\mathbb{R}^{n}} f(x) g(x) d x .
$$

In order to prove our previous statement, we need the following variable version of Minkowski's integral inequality. This result can be found in [5]; however, for the sake of completeness, we include the proof here.

Proposition 2.23. Let $p(\cdot)$ be an exponent function, and $f: \mathbb{R}^{n} \times \mathbb{R}^{n} \rightarrow \mathbb{R}$ be a measurable function.

(a) Suppose that $f(\cdot, y) \in L^{p(\cdot)}$ for a.e. $y \in \mathbb{R}^{n}$ and the mapping $y \rightarrow$ $\|f(\cdot, y)\|_{p(\cdot)}$ is in $L^{1}$. Then

$$
\left\|\int_{\mathbb{R}^{n}} f(\cdot, y) d y\right\|_{p(\cdot)} \leq C \int_{\mathbb{R}^{n}}\|f(\cdot, y)\|_{p(\cdot)} d y,
$$

where $C$ only depends on the bounds of $p(\cdot)$. 
(b) Moreover, for $1<q<p_{-}$, we have

$$
\left\|\left(\int_{\mathbb{R}^{n}}|f(\cdot, y)|^{q} d y\right)^{1 / q}\right\|_{p(\cdot)} \leq C\left(\int_{\mathbb{R}^{n}}\|f(\cdot, y)\|_{p(\cdot)}^{q} d y\right)^{1 / q} .
$$

Proof. By Hölder's inequality and (2.17) we have

$$
\begin{aligned}
\left\|\int_{\mathbb{R}^{n}} f(\cdot, y) d y\right\|_{p(\cdot)} & \leq C \sup _{g:\|g\|_{p^{\prime}(\cdot)} \leq 1}\left[\int_{\mathbb{R}^{n}}\left(\int_{\mathbb{R}^{n}} f(x, y) d y\right)|g(x)| d x\right] \\
& \leq C \sup _{g:\|g\|_{p^{\prime}(\cdot)} \leq 1}\left[\int_{\mathbb{R}^{n}} \int_{\mathbb{R}^{n}} f(x, y)|g(x)| d x d y\right] \\
& \leq C \int_{\mathbb{R}^{n}}\|f(\cdot, y)\|_{p(\cdot)} d y .
\end{aligned}
$$

Now to prove (b), we observe that $\frac{p(\cdot)}{q}$ is an exponent function with $\left(\frac{p(\cdot)}{q}\right)_{-}>1$ and $\|f\|_{p(\cdot)}^{q}=\left\|f^{q}\right\|_{p(\cdot) / q}$ whenever $f \in L^{p(\cdot)}$. Then, from (a) we get

$$
\begin{aligned}
\left\|\left(\int_{\mathbb{R}^{n}}|f(\cdot, y)|^{q} d y\right)^{1 / q}\right\|_{p(\cdot)}^{q} & =\left\|\int_{\mathbb{R}^{n}}|f(\cdot, y)|^{q} d y\right\|_{p(\cdot) / q} \\
& \leq C \int_{\mathbb{R}^{n}}\left\||f(\cdot, y)|^{q}\right\|_{p(\cdot) / q} d y \\
& =C \int_{\mathbb{R}^{n}}\||f(\cdot, y)|\|_{p(\cdot)}^{q} d y
\end{aligned}
$$

as desired.

Proposition 2.24. Let $0<\alpha<n$, and let $p(\cdot)$ be an exponent function with $p_{-}>\frac{n}{\alpha}$ such that $p^{\prime}(\cdot)$ satisfies the doubling condition. If a measurable function $f$ satisfies the pointwise condition

$$
|f(x)-f(y)| \leq C \int_{0}^{2|x-y|} \frac{\left\|\chi_{B(x, t)}\right\|_{p^{\prime}(\cdot)}+\left\|\chi_{B(y, t)}\right\|_{p^{\prime}(\cdot)}}{t^{n-\alpha}} \frac{d t}{t}
$$

for almost all $x, y \in \mathbb{R}^{n}$, then $f \in \mathfrak{L}_{\alpha, p(\cdot)}$.

Proof. Given $r>0$ and $x_{0} \in \mathbb{R}^{n}$, we consider the ball $B=B\left(x_{0}, r\right)$. In order to prove that $f \in \mathfrak{L}_{\alpha, p(\cdot)}$, we will see that

$$
\int_{B}\left|f(x)-m_{B} f\right| d x \leq C|B|^{\alpha / n}\left\|\chi_{2 B}\right\|_{p^{\prime}(\cdot)} .
$$

In fact, as in the proof of Proposition 2.7, from the hypothesis on $f$ and from the fact that $p^{\prime}(\cdot)$ satisfies the doubling condition we get

$$
\begin{aligned}
\int_{B}\left|f(x)-m_{B} f\right| d x & \leq C \int_{B} \int_{0}^{4 r} \frac{\left\|\chi_{B(x, 2 t)}\right\|_{p^{\prime}(\cdot)}}{t^{n-\alpha}} \frac{d t}{t} d x \\
& \leq C \int_{0}^{r} \int_{B}\left\|\chi_{B(x, t)}\right\|_{p^{\prime}(\cdot)} d x \frac{d t}{t^{n-\alpha+1}} .
\end{aligned}
$$


Now, let $q>1$ to be determined later. Applying the Hölder inequality, we have

$$
\begin{aligned}
& \int_{B}\left|f(x)-m_{B} f\right| d x \\
& \leq C|B|^{1 / q^{\prime}} \int_{0}^{r}\left(\int_{B}\left\|\chi_{B(x, t)}\right\|_{p^{\prime}(\cdot)}^{q} d x\right)^{1 / q} \frac{d t}{t^{n-\alpha+1}} \\
& \leq C|B|^{1 / q^{\prime}} \int_{0}^{r}\left(\int_{\mathbb{R}^{n}}\left\|\chi_{B\left(x_{0}, r\right)}(x) \chi_{B(x, t)}(\cdot)\right\|_{p^{\prime}(\cdot)}^{q} d x\right)^{1 / q} \frac{d t}{t^{n-\alpha+1}} .
\end{aligned}
$$

We claim that by taking $q$ such that $\frac{n}{\alpha}<q^{\prime}<p_{-}$we have

$$
\left(\int_{\mathbb{R}^{n}}\left\|\chi_{B\left(x_{0}, r\right)}(x) \chi_{B(x, t)}(\cdot)\right\|_{p^{\prime}(\cdot)}^{q} d x\right)^{1 / q} \leq C t^{n / q}\left\|\chi_{2 B}\right\|_{p^{\prime}(\cdot)}<\infty
$$

for every $0<t<r$. Thus,

$$
\begin{aligned}
\int_{B}\left|f(x)-m_{B} f\right| d x & \leq C\left\|\chi_{2 B}\right\|_{p^{\prime}(\cdot)}|B|^{1 / q^{\prime}} \int_{0}^{r} t^{n / q-n+\alpha-1} d t \\
& =C\left\|\chi_{2 B}\right\|_{p^{\prime}(\cdot)}|B|^{1 / q^{\prime}} r^{\alpha-n / q^{\prime}}=C\left\|\chi_{2 B}\right\|_{p^{\prime}(\cdot)}|B|^{\alpha / n},
\end{aligned}
$$

and so we get our result.

Now, it only remains to prove the claim. In terms of the theory of integration for vector-valued functions, our claim says that $\chi_{B\left(x_{0}, r\right)}(x) \chi_{B(x, t)}(z)$ belongs to the Bochner-Lebesgue space $L_{L^{p^{\prime}(\cdot)}}^{q}$ (see [8], Chap. V.1), whose topological dual space is $L_{L^{p(\cdot)}}^{q^{\prime}}$. Then by duality we can write

$$
\begin{aligned}
& \left\|\chi_{B\left(x_{0}, r\right)}(x) \chi_{B(x, t)}(\cdot)\right\|_{L_{L^{p^{\prime}(\cdot)}}^{q}} \sup _{\| C} \sup _{\left\|g_{t}(x, z)\right\|_{L_{L^{q^{\prime}(\cdot)}}}}\left(\int_{\mathbb{R}^{n}} \int_{\mathbb{R}^{n}} \chi_{B\left(x_{0}, r\right)}(x) \chi_{B(x, t)}(z) g_{t}(x, z) d x d z\right) \\
& \leq C \sup _{\left\|g_{t}(x, z)\right\|_{L_{L^{\prime}}^{q^{\prime}(\cdot)}}}\left(\int_{\mathbb{R}^{n}} \int_{\mathbb{R}^{n}} \chi_{B\left(x_{0}, r\right)}(x) \chi_{B(z, t)}(x) g_{t}(x, z) d x d z\right),
\end{aligned}
$$

where in the last expression we use that $\chi_{B(x, t)}(z)=\chi_{B(z, t)}(x)$. Now, taking into account that for every fixed $0<t<r$, we have $\chi_{B\left(x_{0}, 2 r\right)}(z)=1$ for all $z \in B(x, t)$ whenever $x \in B\left(x_{0}, r\right)$, we get

$$
\begin{aligned}
& \left\|\chi_{B\left(x_{0}, r\right)}(x) \chi_{B(x, t)}(z)\right\|_{L_{L^{p^{\prime}(\cdot)}}^{q}} \\
& \leq C \sup _{\left\|g_{t}(x, z)\right\|_{L_{L^{p(\cdot)}}^{q^{\prime}}} \leq 1}\left(\int_{\mathbb{R}^{n}} \chi_{B\left(x_{0}, 2 r\right)}(z) \int_{\mathbb{R}^{n}} \chi_{B(z, t)}(x)\left|g_{t}(x, z)\right| d x d z\right) \\
& \leq C t^{n / q} \sup _{\left\|g_{t}(x, z)\right\|_{L_{L^{q^{\prime}(\cdot)}}} \leq 1} \int_{2 B}\left\|g_{t}(\cdot, z)\right\|_{q^{\prime}} d z \\
& \leq C t^{n / q}\left\|\chi_{2 B}\right\|_{p^{\prime}(\cdot)} \sup _{\left\|g_{t}(x, z)\right\|_{L_{L^{q^{\prime}(\cdot)}}^{q^{\prime}}} \leq 1}\left\|g_{t}(x, z)\right\|_{L_{L^{q^{\prime}}}^{p(\cdot)}} .
\end{aligned}
$$


Finally, recalling that $\frac{n}{\alpha}<q^{\prime}<p_{-}$, by Proposition 2.23 we conclude that

$$
\begin{aligned}
& \left\|\chi_{B\left(x_{0}, r\right)}(x) \chi_{B(x, t)}(z)\right\|_{L_{L^{p^{\prime}(\cdot)}}^{q}} \sup \leq C t^{n / q}\left\|\chi_{B_{2 R}}\right\|_{p^{\prime}(\cdot)} \operatorname{sg}_{t}(x, z)\left\|_{L_{L^{q^{\prime}(\cdot)}}}\right\| g_{t}(x, z) \|_{L_{L^{p(\cdot)}}^{q^{\prime}}} \\
& \quad \leq C t^{n / q}\left\|\chi_{B_{2 R}}\right\|_{p^{\prime}(\cdot)}<\infty .
\end{aligned}
$$

We note that if, in Proposition 2.24, $p(\cdot)$ is assumed to be constant in the interval $\left(n / \alpha, n /(\alpha-1)^{+}\right)$, then the pointwise condition (2.18) implies that $f$ belongs to the Lipschitz space of order $0<\beta=\alpha-n / p<1$ (see, in addition, [12] and [23]).

In view of Proposition 2.7, we get the following pointwise characterization of $\mathfrak{L}_{\alpha, p(\cdot)}$ without log-Hölder hypotheses on $p(\cdot)$.

Theorem 2.25. Let $0<\alpha<n$, and let $p(\cdot)$ be an exponent function such that $p_{-}>\frac{n}{\alpha}$ and $p^{\prime}(\cdot)$ satisfies the doubling condition. The following conditions are equivalent:

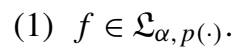

(2) $f$ satisfies (2.18).

Proof. By Proposition 2.24, clearly, (2) implies (1). In order to prove the converse, we proceed in the same way as in Proposition 2.5, but this time considering in (2.2) the properties of $w(x, t)=t^{\alpha-n}\left\|\chi_{B(x, t)}\right\|_{p^{\prime}(\cdot)}$, that is, $p^{\prime}(\cdot)$ satisfies the doubling condition, and $t^{\alpha-n}$ is decreasing.

Theorem 2.26. Let $0<\alpha<n$, and let $p(\cdot)$ be an exponent function such that $p_{-}>\frac{n}{\alpha}$. If $p^{\prime}(\cdot)$ satisfies the doubling condition. Then $\mathfrak{L}_{\alpha, p(\cdot)}=\mathfrak{L}_{\alpha, p(\cdot)}$ for every $1 \leq s<\frac{n}{n-\alpha}$.

Proof. By Hölder's inequality, clearly, $\mathfrak{L}_{\alpha, p(\cdot)} \subset \mathfrak{L}_{\alpha, p(\cdot)}$. On the other hand, if $f \in \mathfrak{L}_{\alpha, p(\cdot)}$, then by Corollary $2.25 f$ satisfies the pointwise estimate (2.18). In view of Proposition 2.7 with $w(x, t)=t^{\alpha-n}\left\|\chi_{B(x, t)}\right\|_{p^{\prime}(\cdot)}$, we only have to prove (2.4). Taking $s$ in $\left(1, \frac{n}{n-\alpha}\right)$, we get

$$
\begin{aligned}
\int_{B}\left(\int_{0}^{r} t^{\alpha-n-1}\left\|\chi_{B(x, t)}\right\|_{p^{\prime}(\cdot)} d t\right)^{s} d x \\
\quad \leq\left(\int_{0}^{r}\left(\int_{B} t^{(\alpha-n-1) s}\left\|\chi_{B(x, t)}\right\|_{p^{\prime}(\cdot)}^{s} d x\right)^{1 / s} d t\right)^{s} \\
\quad=\left(\int_{0}^{r} t^{\alpha-n-1}\left\|\chi_{B\left(x_{0}, r\right)}(x) \chi_{B(x, t)}(z)\right\|_{L_{L^{p^{\prime}(\cdot)}}^{s}} d t\right)^{s} \\
\quad \leq C\left(\int_{0}^{r} t^{\alpha-n-1} t^{n / s}\left\|\chi_{B\left(x_{0}, r\right)}\right\|_{p^{\prime}(\cdot)} d t\right)^{s}
\end{aligned}
$$




$$
\begin{aligned}
& =C\left\|\chi_{B\left(x_{0}, r\right)}\right\|_{p^{\prime}(\cdot)}^{s}\left(\int_{0}^{r} t^{\alpha-n-1+n / s} d t\right)^{s} \\
& =C\left\|\chi_{B\left(x_{0}, r\right)}\right\|_{p^{\prime}(\cdot)}^{s} r^{(\alpha-n) s} r^{n} \\
& =C\left(\left\|\chi_{B}\right\|_{p^{\prime}(\cdot)} r^{(\alpha-n)}\right)^{s} r^{n},
\end{aligned}
$$

where $\alpha-n+\frac{n}{s}>0$. Then $\mathfrak{L}_{\alpha, p(\cdot)}=\mathfrak{L}_{\alpha, p(\cdot)}$ for every $1 \leq s<\frac{n}{n-\alpha}$, as desired.

\section{Fractional Integrals on $\mathbf{B M O}_{w, q}$ Spaces}

In this section, we prove boundedness results for the fractional integral operator. In order to do this, we consider the following definition.

Definition 3.1. Let $w(x, t)$ be a measurable function. We say that $w \in \mathcal{W}_{\infty}$ if there exists a constant $C>0$ such that

$$
\int_{r}^{\infty} \frac{w(x, t)}{t} \frac{d t}{t} \leq C \frac{w(x, r)}{r}
$$

for all $x \in \mathbb{R}^{n}$ and $r>0$.

It should be noticed that condition (3.1) appears in the literature in different contexts. See, for instance, $[12 ; 6]$, and, in the particular case $w(x, t)=$ $t^{\alpha-n}\left\|\chi_{B(x, t)}\right\|_{p^{\prime}(\cdot)}$, our paper [22].

Now, we prove a technical lemma that will be useful in getting one of our main results.

LEMma 3.2. Let $\alpha$ be a real number, and let $w$ be a measurable function satisfying (1.2) and (1.3). If a function $f$ belongs to $B M O_{w, q}$ for a some $1 \leq q<\infty$, then we have

$$
\int_{B} \frac{\left|f(y)-m_{B} f\right|}{|x-y|^{n-\alpha}} d y \leq C\|f\|_{w, q} \int_{0}^{r} \frac{t^{\alpha} w(x, t)}{t} d t,
$$

where $B=B(x, r)$, and $C$ is not dependent on $B$.

Proof. Let $x \in \mathbb{R}^{n}$ and $r>0$. We consider the ball $B=B(x, r)$ and denote $B_{k}=$ $B\left(x, 2^{-k} r\right), k \in \mathbb{N}_{0}$. Then, we estimate

$$
\begin{aligned}
\int_{B} \frac{\left|f(y)-m_{B} f\right|}{|x-y|^{n-\alpha}} d y & =\sum_{k=0}^{\infty} \int_{B_{k}-B_{k+1}} \frac{\left|f(y)-m_{B} f\right|}{|x-y|^{n-\alpha}} d y \\
& \leq C \sum_{k=0}^{\infty}\left(2^{-k} r\right)^{\alpha}\left|B_{k}\right|^{-1} \int_{B_{k}}\left|f(y)-m_{B} f\right| d y \\
& \leq C \sum_{k=0}^{\infty}\left(2^{-k} r\right)^{\alpha} \sum_{j=0}^{k}\left(\left|B_{j}\right|^{-1} \int_{B_{j}}\left|f(y)-m_{B_{j}} f\right|^{q} d y\right)^{1 / q}
\end{aligned}
$$




$$
\begin{aligned}
& \leq C\|f\|_{w, q} \sum_{k=0}^{\infty}\left(2^{-k} r\right)^{\alpha} \sum_{j=0}^{k} w\left(x, 2^{-j} r\right) \\
& =C\|f\|_{w, q} \sum_{j=0}^{\infty}\left(2^{-j} r\right)^{\alpha} w\left(x, 2^{-j} r\right) \\
& \leq C\|f\|_{w, q} \sum_{j=0}^{\infty} \int_{2^{-j-1} r}^{2^{-j} r} t^{\alpha} w(x, t) \frac{d t}{t} \\
& \leq C\|f\|_{w, q} \int_{0}^{r} \frac{t^{\alpha} w(x, t)}{t} d t
\end{aligned}
$$

as desired.

REMARK 3.3. Note that conditions (1.2) and (1.3) are only applied in Lemma 3.2 to get the integral expression involving the function $w(x, t)$. For the particular case $w(x, t)=\Phi(t) t^{-n} \int_{B(x, t)} v(y) d y$, a similar expression can be proved without assuming those hypotheses on the whole $w(x, t)$. In that case we only need to consider the properties of each factor.

Now, for a number $\sigma \geq 0$, we denote $w_{\sigma}(x, t)=t^{\sigma} w(x, t)$ (clearly, $\left.w_{0}=w\right)$. We now prove the main theorem of this section.

THEOREM 3.4. Let $0<\alpha<n$, and letw be a nonnegative measurable function satisfying conditions (1.2), (1.3), and (1.4). Now, if $w_{\alpha} \in \mathcal{W}_{\infty}$, then the fractional integral $I_{\alpha}$ can be extended to a linear bounded operator from $B M O_{w, q}$ into $B M O_{w_{\alpha}, q}$ with $1 \leq q<\infty$ as follows:

$$
\tilde{I}_{\alpha} f(x)=\int_{\mathbb{R}^{n}}\left(\frac{1}{|x-y|^{n-\alpha}}-\frac{1}{|y|^{n-\alpha}}\right) f(y) d y,
$$

so that $\widetilde{I}_{\alpha}$ is well defined on $B M O_{w, q}$.

Proof. First, we prove that the extension of $I_{\alpha}$ to $\widetilde{I}_{\alpha}$ is well defined. For this, we take $f \in \mathrm{BMO}_{w, q}, x \in \mathbb{R}^{n}, r>|x|$, and the ball $B=B(0, r)$. We need to show that $\left|\widetilde{I}_{\alpha} f(x)\right|<\infty$. Since the expression in brackets in (3.3) has zero integral over $\mathbb{R}^{n}$ as a function of $y$, we get

$$
\widetilde{I}_{\alpha} f(x)=\int_{\mathbb{R}^{n}}\left(\frac{1}{|x-y|^{n-\alpha}}-\frac{1}{|y|^{n-\alpha}}\right)\left(f(y)-m_{2 B} f\right) d y=I_{1}(x)+I_{2}(x),
$$

where $I_{1}$ and $I_{2}$ are the integrals over $B(0,2 r)$ and $\mathbb{R}^{n}-B(0,2 r)$, respectively. For $I_{1}$, by Lemma 3.2 and condition (1.2) on $w$ we have

$$
\begin{aligned}
\left|I_{1}(x)\right| & \leq \int_{B(0,2 r)} \frac{\left|f(y)-m_{B(0,2 r)} f\right|}{|y|^{n-\alpha}} d y+\int_{B(0,2 r)} \frac{\left|f(y)-m_{B(0,2 r)} f\right|}{|x-y|^{n-\alpha}} d y \\
& \leq \int_{B(0,2 r)} \frac{\left|f(y)-m_{B(0,2 r)} f\right|}{|y|^{n-\alpha}} d y+\int_{B(x, 4 r)} \frac{\left|f(y)-m_{B(x, 4 r)} f\right|}{|x-y|^{n-\alpha}} d y
\end{aligned}
$$




$$
\begin{aligned}
& +\left|m_{B(x, 4 r)} f-m_{B(0,2 r)} f\right| \int_{B(0,2 r)} \frac{d y}{|x-y|^{n-\alpha}} \\
\leq & C\|f\|_{w, q} \int_{0}^{2 r} \frac{t^{\alpha} w(0, t)}{t} d t+C\|f\|_{w, q} \int_{0}^{4 r} \frac{t^{\alpha} w(x, t)}{t} d t \\
& +C\|f\|_{w, q} w(x, 4 r) r^{\alpha} \\
\leq & C\|f\|_{w, q} r^{\alpha}(w(0,2 r)+w(x, 4 r))<\infty .
\end{aligned}
$$

Now, let us estimate $I_{2}(x)$ for each $x \in B(0, r)$. Applying the mean value theorem, we get

$$
\left|I_{2}(x)\right| \leq C|B|^{1 / n} \int_{\mathbb{R}^{n}-B(0,2 r)} \frac{\left|f(y)-m_{B} f\right|}{|y|^{n-\alpha+1}} d y .
$$

Then, letting $B_{k}=2^{k} B=B\left(0,2^{k} r\right), k \in \mathbb{N}$, we have

$$
\begin{aligned}
\left|I_{2}(x)\right| & \leq C r \sum_{k=1}^{\infty} \int_{B_{k+1}-B_{k}} \frac{\left|f(y)-m_{B} f\right|}{|y|^{n-\alpha+1}} d y \\
& \leq C r \sum_{k=1}^{\infty}\left(2^{k} r\right)^{\alpha-1}\left|B_{k+1}\right|^{-1} \int_{B_{k+1}}\left|f(y)-m_{B} f\right| d y \\
& \leq C r \sum_{k=1}^{\infty}\left(2^{k} r\right)^{\alpha-1} \sum_{j=1}^{k+1}\left(\left|B_{j}\right|^{-1} \int_{B_{j}}\left|f(y)-m_{B_{j}} f\right|^{q} d y\right)^{1 / q} \\
& \leq C\|f\|_{w, q} r \sum_{k=1}^{\infty}\left(2^{k} r\right)^{\alpha-1} \sum_{j=1}^{k+1} w\left(0,2^{j} r\right) \\
& \leq C\|f\|_{w, q} r \sum_{j=1}^{\infty}\left(2^{j} r\right)^{\alpha-1} w\left(0,2^{j} r\right) \\
& \leq C\|f\|_{w, q} r \sum_{j=1}^{\infty} \int_{2^{j} r}^{2^{j+1} r} \frac{t^{\alpha} w(0, t)}{t} \frac{d t}{t} \\
& \leq C\|f\|_{w, q} r \int_{r}^{\infty} \frac{t^{\alpha} w(0, t)}{t} \frac{d t}{t} .
\end{aligned}
$$

Since $w_{\alpha} \in \mathcal{W}_{\infty}$, we conclude that

$$
\left|I_{2}(x)\right| \leq C\|f\|_{w, q} r \frac{r^{\alpha} w(0, r)}{r}=C\|f\|_{w, q} r^{\alpha} w(0, r) .
$$

Finally, from (3.4) and (3.5) we have that $\left|\widetilde{I}_{\alpha} f(x)\right|<\infty$ for all $x \in \mathbb{R}^{n}$.

Let us show the boundedness of the operator $\widetilde{I}_{\alpha}$. To this aim, we observe that from the properties of $w$, for $x \in \mathbb{R}^{n}, r>0$, and $1 \leq q<\infty$, we have

$$
\begin{aligned}
\int_{B(x, r)} & \left(\int_{0}^{r} \frac{t^{\alpha} w(z, t)}{t} d t\right)^{q} d z \\
\leq & \int_{B(x, r)} w(z, r)^{q}\left(\int_{0}^{r} t^{\alpha-1} d t\right)^{q} d z
\end{aligned}
$$




$$
\begin{aligned}
& \leq C w(x, r)^{q} \int_{B(x, r)} r^{\alpha q} d z \\
& \leq C\left(r^{\alpha} w(x, r)\right)^{q} r^{n} .
\end{aligned}
$$

So, this estimate proves that $w_{\alpha}$ satisfies (2.4). Then, by Proposition 2.7, the proof of the theorem will be complete as soon as we prove that for every function $f$ in $\mathrm{BMO}_{w, q}, \widetilde{I}_{\alpha} f$ satisfies a pointwise inequality like (2.1) with $w_{\alpha}$ instead of $w$. In fact, for such a function and given $x_{1}, x_{2}$ points in $\mathbb{R}^{n}$, considering the ball $B=B\left(x_{1}, 2\left|x_{1}-x_{2}\right|\right)$, we have

$$
\begin{aligned}
\left|\widetilde{I}_{\alpha} f\left(x_{1}\right)-\widetilde{I}_{\alpha} f\left(x_{2}\right)\right| & \leq \int_{\mathbb{R}^{n}}\left|\frac{1}{\left|x_{1}-y\right|^{n-\alpha}}-\frac{1}{\left|x_{2}-y\right|^{n-\alpha}}\right|\left|f(y)-m_{B} f\right| d y \\
& =\int_{B}+\int_{\mathbb{R}^{n}-B}=I_{1}+I_{2} .
\end{aligned}
$$

Proceeding in a similar way as in (3.4) and (3.5), we get that

$$
\left|\tilde{I}_{\alpha} f\left(x_{1}\right)-\tilde{I}_{\alpha} f\left(x_{2}\right)\right| \leq C\|f\|_{w, 1} \int_{0}^{2\left|x_{1}-x_{2}\right|} \frac{t^{\alpha} w\left(x_{1}, t\right)+t^{\alpha} w\left(x_{2}, t\right)}{t} d t,
$$

as desired.

Corollary 3.5. Let $\alpha, \beta \in \mathbb{R}^{+}$be such that $0<\alpha+\beta<1$, and let $w(x, t)$ be a nonnegative measurable function satisfying conditions (1.2), (1.3), and (1.4). If $w_{\alpha+\beta} \in \mathcal{W}_{\infty}$, then the fractional integral $I_{\alpha}$ can be extended to a linear bounded operator from $B M O_{w_{\beta}, q}$ to $B M O_{w_{\alpha+\beta}, q}$ with $1 \leq q<\infty$, as in (3.3).

Proof. It is clear that $w_{\beta}(x, t)$ satisfies properties (1.2), (1.3), and (1.4) if $w(x, t)$ does. Then, applying Theorem 3.4 with $w_{\beta}$, we get the result.

REMARK 3.6. It is obvious that $w \equiv 1$ satisfies the hypotheses of the previous corollary. Then the well-known classical results

$$
I_{\alpha}: \mathrm{BMO} \rightarrow \operatorname{Lip}(\alpha)
$$

and

$$
I_{\alpha}: \operatorname{Lip}(\beta) \rightarrow \operatorname{Lip}(\alpha+\beta)
$$

for $\beta>0$ such that $0<\alpha+\beta<1$ are included.

Also, Theorem 3.4 and Corollary 3.5 recover the following results contained in [12] (see Thm. 2.9 and Cor. 2.12):

$$
\begin{gathered}
I_{\alpha}: \operatorname{BMO}(v) \rightarrow \mathrm{BMO}_{\alpha}(v) \quad \text { whenever } v \in H(\alpha, \infty), \\
I_{\alpha}: \mathrm{BMO}_{\beta}(v) \rightarrow \mathrm{BMO}_{\alpha+\beta}(v) \quad \text { whenever } v \in H(\alpha+\beta, \infty),
\end{gathered}
$$

where $H(\alpha, \infty)$ is defined by

$$
|B|^{1 / n-\alpha / n} \int_{\mathbb{R}^{n}-B} \frac{v(y)}{\left|x_{B}-y\right|^{n-\alpha+1}} d y \leq C \frac{1}{|B|} \int_{B} v(y) d y .
$$


In fact, it is easy to see that $v \in H(\alpha, \infty)$ implies that $w(x, t)=t^{\alpha-n} v(B(x, t))$ satisfies (1.3) (see [12]), (1.4), and $\mathcal{W}_{\infty}$ condition. As we have noted in Remark 3.3, although condition (1.2) does not necessarily hold for this particular $w(x, t)$, we get the integral expression appearing in (3.6), that is,

$$
\begin{aligned}
& \left|\tilde{I}_{\alpha} f\left(x_{1}\right)-\tilde{I}_{\alpha} f\left(x_{2}\right)\right| \\
& \quad \leq C\|f\|_{w, 1} \int_{0}^{2\left|x_{1}-x_{2}\right|} \frac{t^{\alpha-n} v\left(B\left(x_{1}, t\right)\right)+t^{\alpha-n} v\left(B\left(x_{2}, t\right)\right)}{t} d t,
\end{aligned}
$$

which, in view of (1.3) (i.e., the doubling condition of the weight $v$ ), gives the following inequality:

$$
\begin{aligned}
\left|\widetilde{I}_{\alpha} f\left(x_{1}\right)-\widetilde{I}_{\alpha} f\left(x_{2}\right)\right| \leq & C\|f\|_{w, 1}\left(\int_{\left|z-x_{1}\right|<2\left|x_{1}-x_{2}\right|} \frac{v(z)}{\left|z-x_{1}\right|^{n-\alpha}} d z\right. \\
& \left.+\int_{\left|z-x_{2}\right|<2\left|x_{1}-x_{2}\right|} \frac{v(z)}{\left|z-x_{2}\right|^{n-\alpha}} d z\right) .
\end{aligned}
$$

By taking the double average over $B$ this clearly implies that $\widetilde{I}_{\alpha} f \in \operatorname{BMO}_{\alpha}(v)$.

\section{The Riesz Transforms}

Let $f$ be a locally integrable function. Recall that for each $j \in\{1, \ldots, n\}$, the Riesz transform operator is given by

$$
\mathrm{R}_{j} f(x)=\lim _{\varepsilon \rightarrow 0^{+}} \int_{|x-y|>\varepsilon} \frac{x_{j}-y_{j}}{|x-y|^{n+1}} f(y) d y .
$$

It is well known that these operators are bounded in weighted $L^{p}$ spaces (see [8] for instance). Moreover, Morvidone [17] proved the boundedness of the Hilbert transform as an operator between certain weighted spaces of functions with mean oscillation controlled by a function $\varphi$, which generalized the results of Muckenhoupt and Wheeden [18] and Peetre [21].

It is important to note that the hypotheses assumed by Morvidone are not contained in ours because that author takes advantage of a better knowledge of the structure of $w(x, t)$ since just a particular case is considered.

Our next theorem gives a similar result for general functions $w(x, t)$.

TheOREm 4.1. Let $1 \leq q<\infty$, and let $w$ be a measurable function satisfying (1.2), (1.3), and (1.4). Suppose that $w \in \mathcal{W}_{\infty}$. Then $\mathrm{R}_{j}$ can be extended to a linear bounded operator $\mathcal{R}_{j} f(x)$ on $\mathrm{BMO}_{w, q}$ as follows:

$$
\mathcal{R}_{j} f(x)=\lim _{\varepsilon \rightarrow 0^{+}} \int_{|x-y|>\varepsilon}\left[\frac{x_{j}-y_{j}}{|x-y|^{n+1}}+\frac{y_{j} \Gamma(y)}{|y|^{n+1}}\right] f(y) d y .
$$

Here $\Gamma(y)$ is the characteristic function of $|y|>1$.

Proof. In view of Theorem 2.13, we have to prove the result only for $1<q<\infty$.

Now, let $1<q<\infty$ and $f \in \mathrm{BMO}_{w, q}$. First, we prove that $\mathcal{R}_{j} f(x)$ is well defined over $\mathrm{BMO}_{w, q}$. It is not difficult to see that $\mathcal{R}_{j} 1=0$. Using this, for each 
$x \in B=B(0, r)$, we get

$$
\begin{aligned}
\mathcal{R}_{j} f(x)= & \mathcal{R}_{j}\left(f-m_{B} f\right)(x) \\
= & \lim _{\varepsilon \rightarrow 0^{+}} \int_{\substack{|x-y|>\varepsilon \\
|y|<2 r}}\left[\frac{x_{j}-y_{j}}{|x-y|^{n+1}}+\frac{y_{j} \Gamma(y)}{|y|^{n+1}}\right]\left(f(y)-m_{B} f\right) d y \\
& +\lim _{\varepsilon \rightarrow 0^{+}} \int_{\substack{|x-y|>\varepsilon \\
|y|>2 r}}\left[\frac{x_{j}-y_{j}}{|x-y|^{n+1}}+\frac{y_{j} \Gamma(y)}{|y|^{n+1}}\right]\left(f(y)-m_{B} f\right) d y \\
= & \mathrm{T}_{1}(x)+\mathrm{T}_{2}(x) .
\end{aligned}
$$

For $\mathrm{T}_{1}$, by the definition of the operator,

$$
\begin{aligned}
\left|\mathrm{T}_{1}(x)\right| \leq & \left|\lim _{\varepsilon \rightarrow 0^{+}} \int_{\substack{|x-y|>\varepsilon \\
|y|<2 r}} \frac{x_{j}-y_{j}}{|x-y|^{n+1}}\left(f(y)-m_{B} f\right) d y\right| \\
& +\lim _{\varepsilon \rightarrow 0^{+}} \int_{\substack{|x-y|>\varepsilon \\
1<|y|<2 r}} \frac{\left|f(y)-m_{B} f\right|}{|y|^{n}} d y<\infty .
\end{aligned}
$$

Since $\left(f(y)-m_{B} f\right) \chi_{2 B} \in L^{q}$, the finiteness a.e. of the first term is a consequence of the boundedness of $\mathrm{R}_{j}$. For the second one, Lebesgue's dominated convergence theorem is applied to get the conclusion.

On the other hand, taking $\varepsilon<r$ in $\mathrm{T}_{2}$, applying the mean value theorem, and considering the increasing sequence of balls $B_{k}=B\left(0,2^{k} r\right), k=1,2, \ldots$, we have

$$
\begin{aligned}
\left|\mathrm{T}_{2}(x)\right| & \leq C r \int_{|y|>2 r} \frac{\left|f(y)-m_{B} f\right|}{|x-y|^{n+1}} d y \\
& \leq C r \sum_{k=1}^{\infty} \int_{2^{k} r<|y|<2^{k+1} r} \frac{\left|f(y)-m_{B} f\right|}{|y|^{n+1}} d y \\
& \leq C \sum_{k=1}^{\infty} \frac{1}{2^{k}} \frac{1}{\left|B_{k+1}\right|} \int_{B_{k+1}}\left|f(y)-m_{B} f\right| d y \\
& \leq C \sum_{k=1}^{\infty} \sum_{j=1}^{k+1} \frac{1}{2^{k}}\left(\frac{1}{\left|B_{j}\right|} \int_{B_{j}}\left|f(y)-m_{B j} f\right|^{q} d y\right)^{1 / q} \\
& =C\|f\|_{w, q} \sum_{j=1}^{\infty} w\left(0,2^{j} r\right) \int_{2^{j} r}^{2^{j+1} r} \frac{1}{2^{j}} \frac{d t}{t} \\
& \leq C\|f\|_{w, q} r \int_{r}^{\infty} \frac{w(0, t)}{t} \frac{d t}{t} \\
& \leq C\|f\|_{w, q} w(0, r)<\infty,
\end{aligned}
$$

where the last inequality holds because $w \in \mathcal{W}_{\infty}$. Finally, taking a sequence $\left\{B_{n}\right\}$ of balls such that $B_{n} \nearrow \mathbb{R}^{n}$ and applying the previous reasoning for each $B_{n}$, we get that $\mathcal{R}_{j} f$ is finite a.e. 
Now, we prove the boundedness of the operator acting on $\mathrm{BMO}_{w, q}$. As for (4.2), we consider $g=f-m_{B} f, g_{1}=g \chi_{2 B}$, and $g_{2}=g-g_{1}$ with given $B=$ $B\left(x_{0}, r\right)$.

First, we study $R_{j} g_{1}$. From the boundedness of the classical Riesz transform we have

$$
\begin{aligned}
\int_{B}\left|\mathcal{R}_{j} g_{1}(x)-m_{B}\left(\mathcal{R}_{j} g_{1}\right)\right|^{q} d x & =\int_{B}\left|\mathrm{R}_{j} g_{1}(x)-m_{B}\left(\mathrm{R}_{j} g_{1}\right)\right|^{q} d x \\
& \leq 2^{q} \int_{B}\left|\mathrm{R}_{j} g_{1}(x)\right|^{q} d x \\
& \leq C \int_{2 B}\left|f(x)-m_{B} f\right|^{q} d x \\
& \leq C\|f\|_{w, q}^{q} w\left(x_{0}, 2 r\right)^{q}|B| .
\end{aligned}
$$

This and (1.3) allow us to conclude that

$$
\frac{1}{w\left(x_{0}, r\right)}\left(\frac{1}{|B|} \int_{B}\left|\mathcal{R}_{j} g_{1}(x)-m_{B}\left(\mathcal{R}_{j} g_{1}\right)\right|^{q} d x\right)^{1 / q} \leq C\|f\|_{w, q} .
$$

On the other hand, for $R_{j} g_{2}$, taking $\varepsilon<r$ and then applying the mean value theorem, by the same reasoning as used for (4.3) we have

$$
\begin{aligned}
\left|\mathcal{R}_{j} g_{2}(x)-\mathcal{R}_{j} g_{2}(z)\right| & \leq \int_{\mathbb{R}^{n}-2 B}\left|\frac{x_{j}-y_{j}}{|x-y|^{n+1}}+\frac{z_{j}-y_{j}}{|z-y|^{n+1}}\right|\left|f(y)-m_{B} f\right| d y \\
& \leq C r \int_{\mathbb{R}^{n}-2 B} \frac{\left|f(y)-m_{B} f\right|}{\left|x_{0}-y\right|^{n+1}} d y \\
& \leq C\|f\|_{w, q} r \int_{r}^{\infty} \frac{w\left(x_{0}, t\right)}{t} \frac{d t}{t} \\
& \leq C\|f\|_{w, q} w\left(x_{0}, r\right)<\infty .
\end{aligned}
$$

Finally, by Hölder's inequality we can write

$$
\begin{aligned}
\int_{B}\left|\mathcal{R}_{j} g_{2}(x)-m_{B}\left(\mathcal{R}_{j} g_{2}\right)\right|^{q} d x & \leq \frac{1}{|B|} \int_{B} \int_{B}\left|\mathcal{R}_{j} g_{2}(x)-\mathcal{R}_{j} g_{2}(z)\right|^{q} d z d x \\
& \leq C\|f\|_{w, q}^{q} \frac{1}{|B|} \int_{B} \int_{B} w\left(x_{0}, r\right)^{q} d z d x \\
& =C\|f\|_{w, q}^{q}|B| w\left(x_{0}, r\right)^{q} .
\end{aligned}
$$

So the theorem is proved.

REMARK 4.2. For $w(x, t)=\phi(t)$, the condition $\phi \in \mathcal{W}_{\infty}$ implies that $\phi$ is of upper type $\beta$ with $\beta<1$, as it is proved in Lemma (3.3) of [12], which in particular establishes that $\phi$ satisfies (1.3). Hence, we can prove that if $\phi$ is a nonnegative and nondecreasing function such that $\phi \in \mathcal{W}_{\infty}$, then $\mathcal{R}_{j}$ can be extended to a bounded linear operator on $\mathrm{BMO}_{\phi}$. This last result is contained in [21]. 


\section{References}

[1] P. Acquistapace, On BMO regularity for lineal elliptic systems, Ann. Mat. Pura Appl. (4) CLXI (1992), 231-269.

[2] M. Bramanti and L. Brandolini, Estimates of BMO type for singular integrals on spaces of homogeneous type and applications to hypoelliptic PDEs, Rev. Mat. Iberoam. 21 (2005), 511-556.

[3] S. Campanato, Propietà di hölderianità di alcune classi di funzioni, Ann. Sc. Normale. Sup. Pisa 17 (1963), 175-188.

[4] D. Cruz-Uribe, A. Fiorenza, and C. J. Neugebauer, Weighted norm inequalities for the maximal operator on variable Lebesgue spaces, J. Math. Anal. Appl. 394 (2012), no. 2, 744-760.

[5] D. Cruz-Uribe and A. Fiorenza, Variable Lebesgue spaces. Foundations and harmonic analysis, p. x+312, Birkhäuser/Springer, Heidelberg, 2013.

[6] D. Cruz-Uribe, L. Forzani, and D. Maldonado, The structure of increasing weights on the real line, Houston J. Math. 34 (2008), no. 3, 951-983.

[7] L. Diening, P. Harjulehto, P. Hästö, and M. Růžička, Lebesgue and Sobolev spaces with variable exponents, Lecture Notes in Math., 2017, Springer, Heidelberg, 2011.

[8] J. García-Cuerva and J. L. Rubio de Francia, Weighted norm inequalities and related topics, North-Holland Mathematics Studies, 116, North Holland, Amsterdam, 1985.

[9] X. Fan and D. Zhao, On the spaces $L^{p(x)}(\Omega)$ and $W^{m, p(x)}(\Omega)$, J. Math. Anal. Appl. 263 (2001), no. 2, 424-446.

[10] B. Franchi, C. Pérez, and R. L. Wheeden, Self-improving properties of JohnNirenberg and Poincaré inequalities on spaces of homogeneous type, J. Funct. Anal. 153 (1998), no. 1, 108-146.

[11] P. Harjulehto, P. Hästö, and M. Pere, Variable exponent Lebesgue spaces on metric spaces: the Hardy-Littlewood maximal operator, Real Anal. Exchange 30 (2004/2005), no. 1, 87-103.

[12] E. Harboure, O. Salinas, and B. Viviani, Boundedness of the fractional integral on weighted Lebesgue and Lipschitz spaces, Trans. Amer. Math. Soc. 349 (1997), no. 1, 235-255.

[13] , Relations between weighted Orlicz and $B M O_{\Phi}$ spaces through fractional integrals, Comment. Math. Univ. Carolin. 40 (1999), 53-69.

[14] S. Janson, On functions with conditions on the mean oscillation, Ark. Math. 14 (1976), 189-196.

[15] O. Kováčik and J. Rákosník, On spaces $L^{p(x)}$ and $W^{k, p(x)}$, Czechoslovak Math. J. 41(116) (1991), no. 4, 592-618.

[16] G. N. Meyer, Mean oscillation over cubes and Hölder continuity, Proc. Amer. Math. Soc. 15 (1964), 717-721.

[17] M. Morvidone, Weighted $\mathrm{BMO}_{\phi}$ spaces and the Hilbert transform, Rev. Un. Mat. Argentina 44 (2003), no. 1, 1-16.

[18] B. Muckenhoupt and R. L. Wheeden, Weighted bounded mean oscillation and the Hilbert transform, Studia Math. T. LIV (1976), 221-237.

[19] E. Nakai and K. Yabuta, Pointwise multipliers for functions of bounded mean oscillation, J. Math. Soc. Japan 37 (1985), 207-218.

[20] E. Nakai, Pointwise multipliers for functions of weighted bounded mean oscillation, Studia Math. 105 (1993), 105-119.

[21] J. Peetre, On the theory of $\mathfrak{L}_{p, \lambda}$ spaces, J. Funct. Anal. 4 (1969), 71-87.

[22] M. Ramseyer, O. Salinas, and B. Viviani, Lipschitz type smoothness of the fractional integral on variable exponent spaces, J. Math. Anal. Appl. 403 (2013), no. 1, 95-106. 
[23] S. Spanne, Some function spaces defined using the mean oscillation over cubes, Ann. Sc. Normale. Sup. Pisa 19 (1965), 593-607.

\section{Ramseyer}

Instituto de Matemática Aplicada del Litoral CONICET-UNL and

Facultad de Ingeniería Química

Universidad Nacional del Litoral

Santa Fe

Argentina

mramseyer@santafe-conicet.gov.ar
O. Salinas

Instituto de Matemática Aplicada del Litoral CONICET-UNL and Facultad de Ingeniería Química Universidad Nacional del Litoral Santa Fe

Argentina

salinas@santafe-conicet.gov.ar

B. Viviani

Instituto de Matemática Aplicada del Litoral CONICET-UNL and

Facultad de Ingeniería Química

Universidad Nacional del Litoral

Santa Fe

Argentina 